\title{
Intercostal vessel screening prior to pleural interventions by the respiratory physician: a prospective study of real world practice
}

\author{
Eihab 0. Bedawi (1) ${ }^{1,2,3}$, Ambika Talwar ${ }^{1}$, Maged Hassan (10 ${ }^{1,2,4}$, \\ David J. McCracken ${ }^{1}$, Rachelle Asciak ${ }^{1,2}$, Rachel M. Mercer ${ }^{1,2}$, \\ Nikolaos I. Kanellakis ${ }^{1,2,3,5}$, Fergus V. Gleeson ${ }^{1,6}$, Rob J. Hallifax ${ }^{1,2}$, \\ John M. Wrightson ${ }^{1,2}$ and Najib M. Rahman ${ }^{1,2,3,5}$
}

Affiliations: ${ }^{1}$ Oxford Centre for Respiratory Medicine, Oxford University Hospitals NHS Foundation Trust, Oxford, UK. ${ }^{2}$ Oxford Respiratory Trials Unit, University of Oxford, Oxford, UK. ${ }^{3}$ NIHR Biomedical Research Centre, University of Oxford, Oxford, UK. ${ }^{4}$ Chest Diseases Dept, Faculty of Medicine, Alexandria University, Alexandria, Egypt. ${ }^{5}$ Laboratory of Pleural Translational Research, Nuffield Dept of Medicine, University of Oxford, Oxford, UK. ' Dept of Radiology, Churchill Hospital, Oxford University Hospitals NHS Trust, Oxford, UK.

Correspondence: Eihab O. Bedawi, Oxford Respiratory Trials Unit, Churchill Hospital, Old Road, Oxford OX3 7LE, UK. E-mail: eihabbedawidgmail.com

@ERSpublications

This study demonstrates that, as the scope of physicians' pleural practices widens, it is feasible for respiratory physicians to routinely detect the intercostal vessels using the same low frequency transducer when conducting procedures http://bit.ly/2S3SeYo

Cite this article as: Bedawi EO, Talwar A, Hassan M, et al. Intercostal vessel screening prior to pleural interventions by the respiratory physician: a prospective study of real world practice. Eur Respir J 2020; 55: 1902245 [https://doi.org/10.1183/13993003.02245-2019].

\section{ABSTRACT}

Introduction: The rising incidence of pleural disease is seeing an international growth of pleural services, with physicians performing an ever-increasing volume of pleural interventions. These are frequently conducted at sites without immediate access to thoracic surgery or interventional radiology and serious complications such as pleural bleeding are likely to be under-reported.

Aim: To assess whether intercostal vessel screening can be performed by respiratory physicians at the time of pleural intervention, as an additional step that could potentially enhance safe practice.

Methods: This was a prospective, observational study of 596 ultrasound-guided pleural procedures conducted by respiratory physicians and trainees in a tertiary centre. Operators did not have additional formal radiology training. Intercostal vessel screening was performed using a low frequency probe and the colour Doppler feature. Results: The intercostal vessels were screened in $95 \%$ of procedures and the intercostal artery (ICA) was successfully identified in $53 \%$ of cases. Screening resulted in an overall site alteration rate of $16 \%$ in all procedures, which increased to $30 \%$ when the ICA was successfully identified. This resulted in procedure abandonment in $2 \%$ of cases due to absence of a suitable entry site. Intercostal vessel screening was shown to be of particular value in the context of image-guided pleural biopsy.

Conclusion: Intercostal vessel screening is a simple and potentially important additional step that can be performed by respiratory physicians at the time of pleural intervention without advanced ultrasound expertise. Whether the widespread use of this technique can improve safety requires further evaluation in a multi-centre setting with a robust prospective study. 


\section{Introduction}

It is now widely accepted amongst physicians that thoracic ultrasound (TUS) significantly improves success rates and reduces complications of pleural procedures. Its use is highlighted in major guidelines [1] and forms an essential component of training curricula for respiratory physicians. The scope of physician-based TUS and pleural services varies between centres and includes diagnostic and therapeutic aspiration, intercostal drain insertion, indwelling pleural catheter insertion, local anaesthetic thoracoscopy and image-guided pleural biopsy. Importantly, the number of these services is growing worldwide to meet an increasing patient need and, subsequently, so is the volume of pleural interventions that are being conducted by physicians.

The risk and potential complications associated with these procedures can sometimes be taken for granted, particularly in the context of diagnostic aspiration (the famous "how much damage can one do with a green needle?"). However, one must remember that these procedures involve penetration of a major body cavity and potential complications include skin and pleural space infection, damage to surrounding organs, pneumothorax, vasovagal events and bleeding due to intercostal artery (ICA) laceration. It is also worth noting that these procedures are often conducted in centres without on-site access to a thoracic surgeon.

Injury to the ICA is an infrequent complication but the combination of a large potential pleural cavity, negative intrapleural pressure and inaccessibility to external compression means that bleeding associated with this can be life threatening [2-4] and frequently requires repair by invasive procedures [5-7]. Pleural haemorrhage is reported to occur in up to $2 \%$ of thoracenteses, up to $13 \%$ of intercostal drain insertions and up to $4 \%$ of thoracoscopies [8]. Retrospective case series and a previous national survey of complications [9] suggest that these figures are likely to be higher and limited by the general under-reporting of complications in the literature.

Whilst operator experience and the use of TUS to guide pleural intervention may reduce risk of pneumothorax and visceral injury, it cannot protect against risk of intrapleural haemorrhage from ICA laceration without further specific sonographic assessment using colour Doppler. The ability of this mode to image the ICA and collaterals has been established $[10,11]$ and its use in pleural intervention has been supported [12]. However, Doppler ultrasound has, to date, not been studied in a real world population about to undergo pleural intervention and this may be why it does not appear to be widely acknowledged and remains absent from current guidelines [1].

When planning pleural procedures, current guidelines advocate avoiding a posterior approach wherever possible and instead targeting the most lateral aspect of the "safe triangle" (where the ICA is thought to be more protected by the inferior edge of the superior rib). However, computed tomography (CT) studies have shown that the ICA can take a variable course. Whilst the risk of the ICA being exposed is greater in the first $6 \mathrm{~cm}$ paraspinally, a more lateral position is not entirely protective [13]. Older patients and more cephalad rib spaces tend to be associated with a highly variable ICA position laterally [13].

Given the potential for TUS to enable ICA identification at the time of intervention, we aimed to evaluate its utility in routine pleural intervention within a high volume tertiary pleural service.

\section{Methods}

Data was collected prospectively at two different time-points over a 3-year period (2015-2018) for all pleural interventions undertaken by physicians and supervised trainee physicians in our pleural unit. A total of 404 procedures were carried out over the initial 12 month evaluation period between July 2015 and July 2016 [14]. A further 192 procedures were analysed between January and May 2018 and added to the dataset in order to assess for effects over time.

As part of routine clinical care our service records, a standard dataset for all pleural interventions, included: 1) the intended site of the procedure (and hence the site of ICA screening); 2) whether ICA screening was attempted; 3) whether the ICA was successfully identified; and 4) whether this had any influence on the procedure site (i.e. no effect, site altered, or procedure abandoned).

\section{Scanning technique}

ICA screening became a routine part of our pleural intervention practice in 2015 and data collection began at around the same time. The technique was devised by the unit's lead physician (N.M. Rahman) and a standard operating procedure was constructed for all operators. This involved identifying a suitable site for pleural intervention using TUS and interrogation of the intercostal space at that level for the ICA.

The patient is positioned in the sitting or lateral decubitus position (the latter would be adopted as standard for all medical thoracoscopy, image-guided biopsy and indwelling pleural catheter procedures, as 
well as occasionally for other procedures in more unwell patients. The operator would begin by scanning the "normal" hemithorax to ensure that no new abnormalities had developed since previous examination. This would then be followed by identification of the kidney, liver/spleen and diaphragm on the side of the planned procedure before proceeding to scan the hemithorax of interest, positioning it and the ultrasound screen in the same visual plane (regardless of whether the patient is sitting or in the lateral decubitus position).

The routine scanning technique in the unit is using the low frequency $(2-5 \mathrm{~Hz})$ curvilinear probe slowly interrogating each intercostal space whilst scanning along the intercostal plane (rather than in the longitudinal plane with the transducer indicator in a cephalad position), as this allows superior visualisation of the pleura and peripheral lung tumours, as well as the consolidated lung [15]. The most suitable intercostal space is chosen based on it being as lateral as possible and containing the largest pocket of fluid, as well as the absence of any organ. Particular attention is paid to the diaphragm, which is visualised during a full breathing cycle.

The colour Doppler function is then switched on and the Doppler box is adjusted to cover the region of interest, including all layers from the skin to approximately $2 \mathrm{~cm}$ below the parietal pleura. The Doppler receiver gain is adjusted by rotating the colour flow knob to the point of minimum Doppler artefact (minimal colour flow demonstrated in the pleural space). The scale of the colour Doppler is standardised at +18.2 to $-18.2 \mathrm{~cm} \cdot \mathrm{s}^{-1}$ as per standard factory abdominal probe settings.

Colour Doppler is then applied using the same low frequency curvilinear probe, to angulate across the intended intercostal space, slowly sweeping from the inferior rib shadow to the superior rib shadow along the intercostal plane (see figure 1) whilst assessing for the presence of the intercostal vessels.

Where an ICA (or vein, with the assumption that the artery is within neighbouring proximity) is detected via Doppler, a decision is made by the operator as to whether its presence is in the field of the intended intervention. The operator could then choose to alter the procedure site (normally adjusting $1-2 \mathrm{~cm}$ laterally) and record this, or make no alteration and proceed as planned. Alternatively, if it is deemed that there is no suitable intervention site due to the presence of intercostal vessels, the procedure can be abandoned completely. Only vessels that are consistent with a true intercostal anatomical location (figure 2) are considered, as even though subcutaneous vessels are frequently identified, bleeding complications from these are minor and can be easily treated with compression.

All the TUS scans were conducted using the unit's HI VISION Avius Ultrasound machine (Hitachi Ltd, Tokyo, Japan) located in our pleural intervention suite, under the standard "dim light" environment used during all procedures.

\section{Statistical analysis}

The total dataset of 596 procedures was analysed in combination (for overall detection ability and confidence intervals (CIs)) and separately by time period (to add consistency and provide external validity). No pre-hoc sample size calculations were conducted (as no outcome change was considered) and procedures undertaken by radiologists were not included. Rates of screening, identification and site
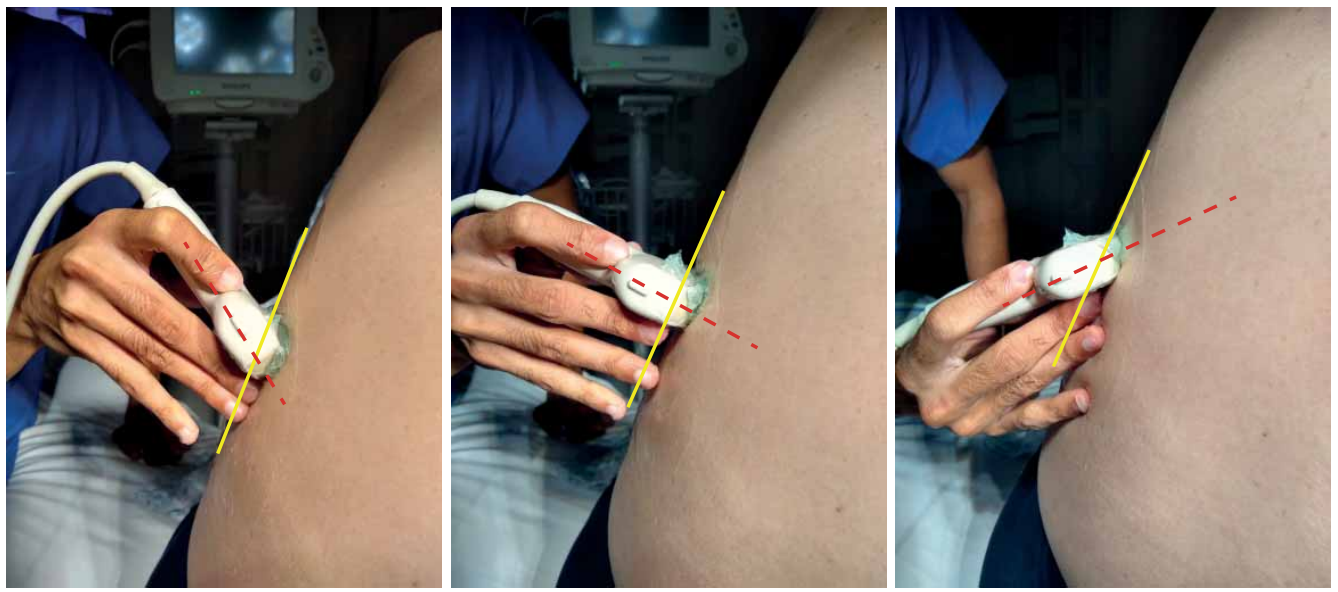

FIGURE 1 Interrogation of the intercostal vessels using a "fanning" technique through different angles of the intercostal space. 


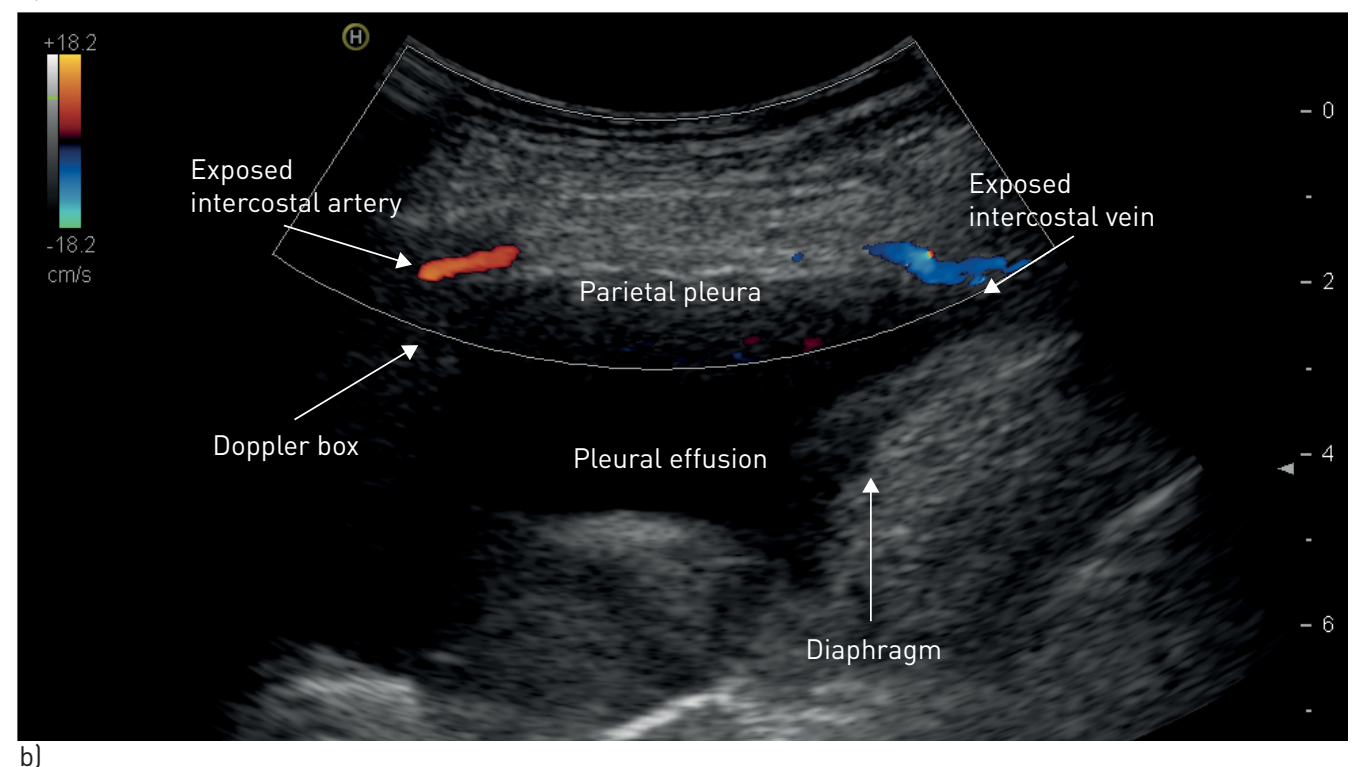

b)

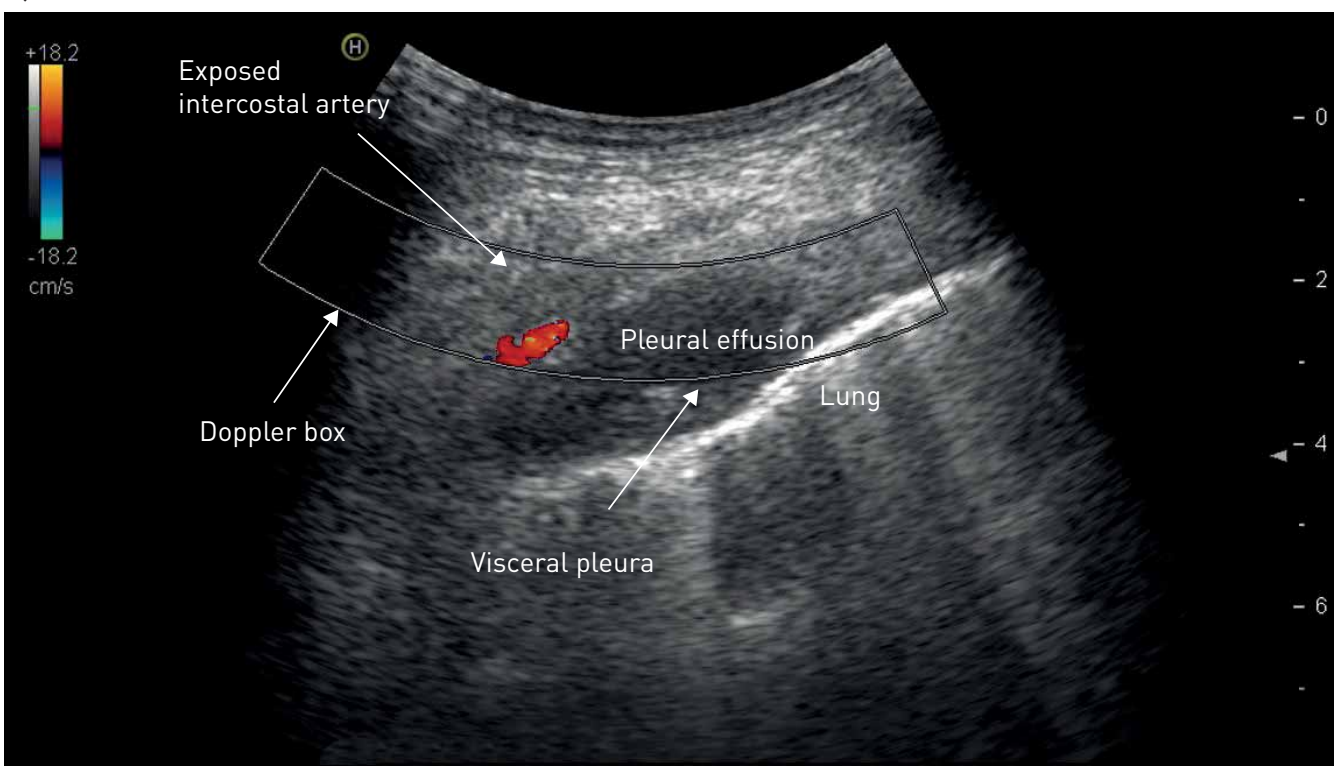

FIGURE 2 Doppler ultrasound screening of the intercostal vessels prior to intervention in two separate patients al and b).

alteration were then compared (as subgroup analyses) based on procedure and site of procedure. Chi-squared statistics were used for categorical variables.

\section{Results}

In the combination dataset, ICA identification was attempted in 563 out of 596 procedures (94\%) and identification at the site of proposed pleural intervention was successful in 298 out of these 563 cases (52.9\%). Detection of the ICA led to procedure site alteration in 88 out of the 298 procedures $(29.5 \%)$ where it was detected, which equates to an overall site alteration rate of $15.6 \%$ when ICA identification was attempted. In seven out of 298 procedures $(2.3 \%)$, the ICA was identified in all rib spaces at potentially suitable intervention sites, leading to no procedure being undertaken. This equates to an overall procedure abandonment rate of $1.2 \%$ when ICA identification is attempted (figure 3 ).

\section{Details of abandoned procedures}

Abandoned procedures were as follows: 1) two diagnostic aspirations for suspected pleural infection with small posterior collections (see figures $4 \mathrm{a}$ and $4 \mathrm{~b}$ ) (treated empirically with antibiotics and monitored 


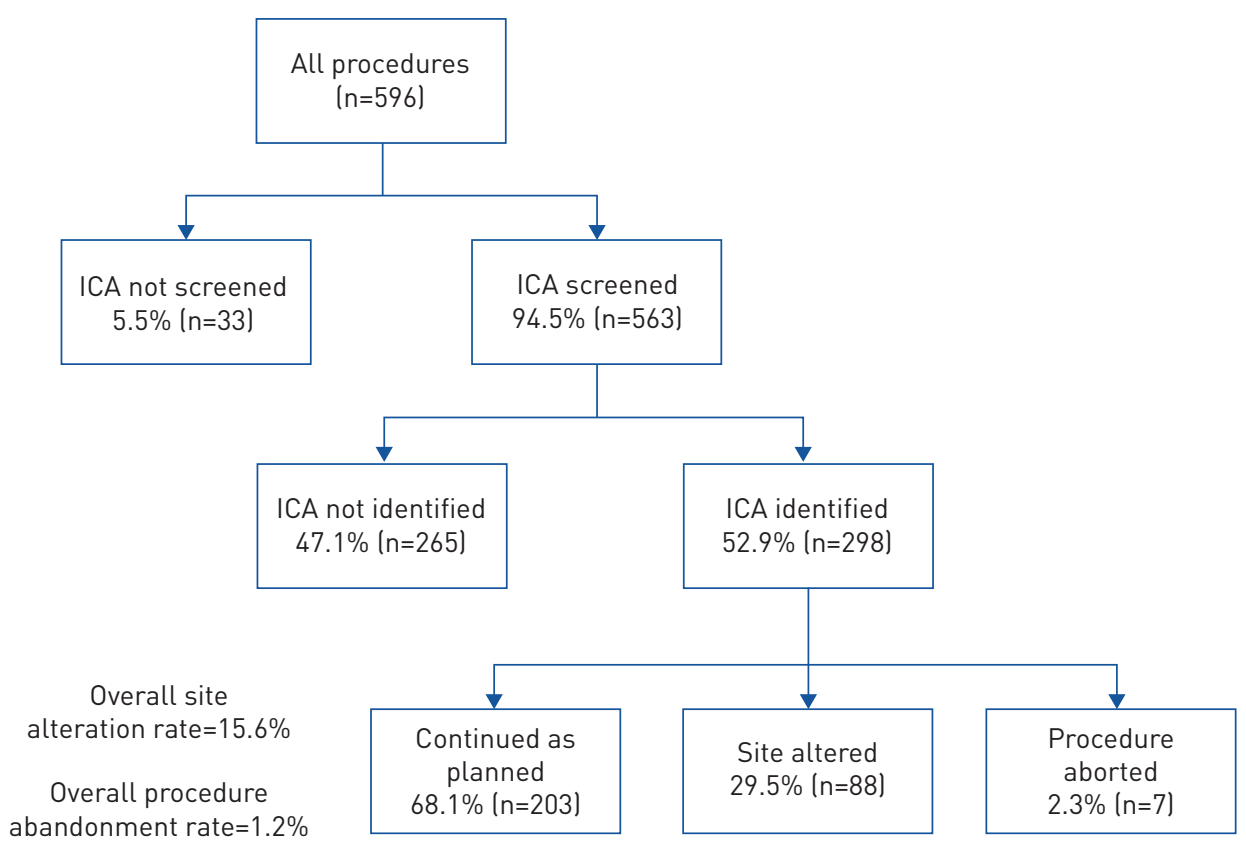

FIGURE 3 Flow chart summarising the main results of screening. ICA: intercostal artery.

with ultrasound); 2) one attempted chest-drain insertion in a case of confirmed pleural infection (treated with antibiotics alone, subsequently required referral for VATS decortication); 3) three ultrasound-guided pleural biopsies (two sent for CT-guided biopsy of an alternative site, one converted to diagnostic aspiration alone); and 4) one planned thoracoscopy for a case of persistent cytology-negative exudative effusion in the context of likely rheumatoid pleuritis but with clinical features for warranting exclusion of malignancy (smoker, weight loss, previous malignancy).

\section{Subgroup analysis}

Comparison between time periods

Analysing the datasets separately over time, baseline characteristics were well matched for all parameters (see table 1).

\section{Comparison by site of procedure}

The dataset was analysed by procedure site to determine how much influence it had on ICA identification. Results are shown in table 2. Only one thoracoscopy procedure was conducted in the anterior axillary line (AAL) and the ICA was not identified.

a)

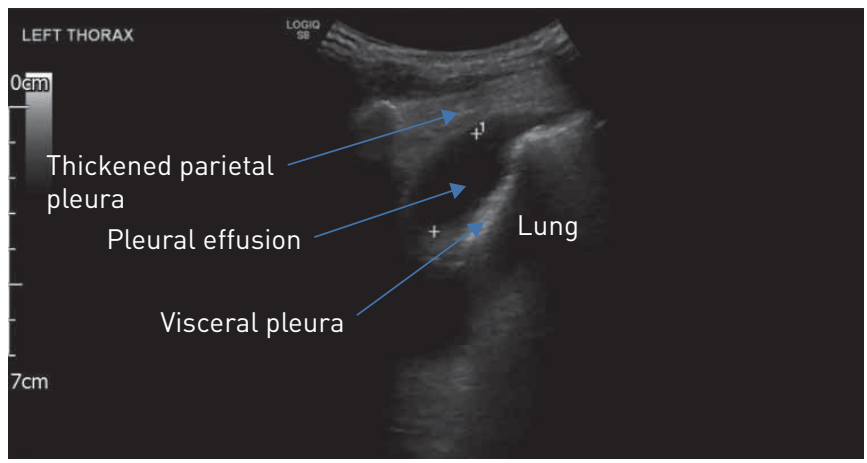

b)

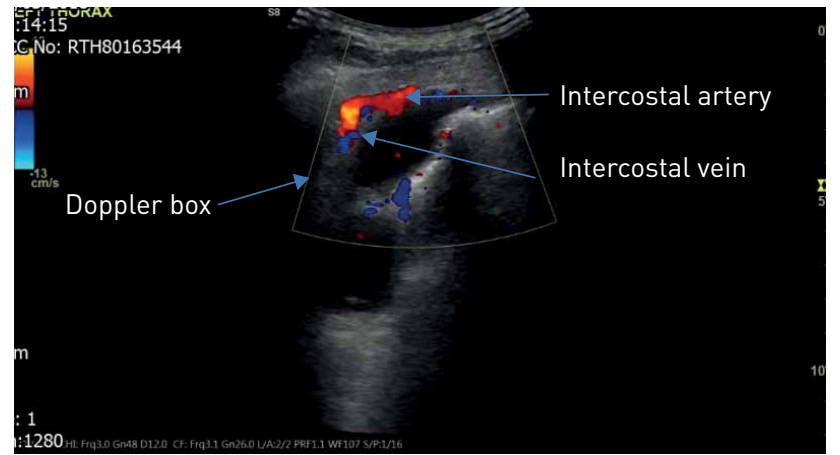

FIGURE 4 a) Small posterior pleural effusion for intended diagnostic aspiration and b) procedure abandoned following Doppler identification of intercostal artery (ICA). 
TABLE 1 Comparison of data collected between defined time periods

\begin{tabular}{lccc} 
& $\mathbf{2 0 1 5}-\mathbf{2 0 1 6}$ (n=404) & 2018 (n=192) & p-value \\
\hline Data period & July 01, 2015-July 31, 2016 & January 01-May 30, 2018 & \\
Male & $257(63.6)$ & $121(63.0)$ & 0.888 \\
Female & $147(36.4)$ & $71(37.0)$ & \\
Age years median & 69.0 & 70.2 & 0.79 \\
ICA attempted & $386(95.5)$ & $177(92)$ & 0.94 \\
ICA identified & $192(49.7)$ & $106(55.2)$ & 0.80 \\
Site altered & $56(13.8)$ & $32(15.0)$ & 0.81 \\
Procedure abandoned & $5(1.2)$ & $2(1.0)$ & 0.84 \\
\hline
\end{tabular}

Data are presented as $\mathrm{n}(\%)$ unless otherwise stated. ICA: intercostal artery.

TABLE 2 Rates of intercostal artery (ICA) identification by procedure site

\begin{tabular}{lcc} 
Site & Proportion of procedures & ICA identified \\
\hline Posterior & 7.8 & 100 \\
PAL & 57.2 & 51 \\
MAL & 34.9 & 42
\end{tabular}

Data are presented as \%. PAL: posterior axillary line; MAL: mid-axillary line.

Comparison by procedure

We found that the rate of successful identification of an ICA was significantly higher for medical thoracoscopy, image-guided biopsy and diagnostic aspiration (table 3). Additionally, it was significantly more likely for ICA identification to result in alteration of the intended site of intervention when performing image-guided biopsies (table 4).

\section{Training and competence}

Ten of the 13 operators (77\%) involved in the procedures in our dataset were either respiratory trainees or pre-Certificate of Completion of Training (CCT) pleural fellows with Royal College of Radiologists (RCR UK) Level 1 TUS competency and no additional formal ultrasound training. The remaining three operators were

TABLE 3 Rates of intercostal artery (ICA) screening and of successful identification

\begin{tabular}{lccccc} 
Procedure & $\begin{array}{c}\text { Procedures } \\
\text { performed }\end{array}$ & $\begin{array}{c}\text { ICA screenings } \\
\text { attempted }\end{array}$ & $\begin{array}{c}\text { Successful } \\
\text { identification }\end{array}$ & $\begin{array}{c}\text { Chi-squared (one } \\
\text { degree of freedom) }\end{array}$ & p-value \\
\hline $\begin{array}{c}\text { Medical } \\
\text { thoracoscopy }\end{array}$ & $105(17.6)$ & $100(95.2)$ & $41(41.0)$ & 6.95 & $<0.01$ \\
$\begin{array}{c}\text { Image-guided } \\
\text { biopsy }\end{array}$ & $72(12)$ & $70(97.2)$ & $51(72.9)$ & 12.74 & $<0.01$ \\
$\begin{array}{c}\text { Intercostal drain } \\
\text { insertion }\end{array}$ & $62(10.4)$ & $54(87.1)$ & $29(53.7)$ & 0.01 & 0.905 \\
$\begin{array}{c}\text { Indwelling pleural } \\
\text { catheter }\end{array}$ & $47(7.9)$ & $46(97.9)$ & $19(41.3)$ & 3.22 & 0.728 \\
$\begin{array}{c}\text { Therapeutic } \\
\text { aspiration }\end{array}$ & $256(42.9)$ & $245(95.7)$ & $126(51.4)$ & 0.34 & 0.560 \\
$\begin{array}{c}\text { Diagnostic } \\
\text { aspiration }\end{array}$ & $54(9.1)$ & $48(88.9)$ & $32(66.7)$ & 7.71 & $<0.01$ \\
All procedures & 596 & $563(94)$ & $298(52.9)$ & & \\
\hline
\end{tabular}

Data are presented as $\mathrm{n}$ or $\mathrm{n}(\%)$. $\mathrm{p}$-Values in bold are statistically significant. \#: procedures performed, where the percentage is the proportion of all procedures; I: ICA screenings attempted, where the percentage is the proportion of procedures performed; ${ }^{+}$: ICAs successfully identified, where the percentage is the proportion of screenings attempted. 


\begin{tabular}{lcccc} 
TABLE 4 Rates of intervention site alteration when an intercostal artery (ICA) was identified \\
Procedure & $\begin{array}{c}\text { ICA } \\
\text { identified }\end{array}$ & Site altered ${ }^{\#}$ & $\begin{array}{c}\text { Chi-squared (one degree } \\
\text { of freedom) }\end{array}$ & p-value \\
\hline Medical thoracoscopy & 41 & $13(31.7)$ & 0.11 & 0.742 \\
Image-guided biopsy & 51 & $27(52.9)$ & 16.20 & $<0.01$ \\
Intercostal drain insertion & 29 & $8(27.6)$ & 0.06 & 0.809 \\
Indwelling pleural catheter & 19 & $3(15.8)$ & 1.84 & 0.175 \\
Therapeutic aspiration & 126 & $30(23.8)$ & 3.43 & 0.064 \\
Diagnostic aspiration & 32 & $7(21.9)$ & 1.01 & 0.315 \\
All procedures & 298 & $88(29.5)$ & &
\end{tabular}

Data is presented as $\mathrm{n}$ or $\mathrm{n}(\%)$. $\mathrm{p}$-Values in bold are statistically significant. " : sites altered, where the percentage is the proportion of ICAs identified.

senior fellow or consultant (attending) level doctors with Level $2 / 3$ ultrasound competency. As per local standard operating procedures, a Level $2 / 3$ operator would be required in the intervention suite (in a training/ supervisory capacity) for all medical thoracoscopy and image-guided biopsy procedures, but would not routinely be present for other procedures. For these advanced procedures, they would directly observe the intercostal screening scan to ensure correct documentation of presence or absence of the intercostal vessels at the site of procedure. Throughout the periods studied, our unit had one intra-pleural bleed following image-guided biopsy (ICA screening attempted but ICA not identified), which was managed conservatively with external pressure and a chest tube. This equates to a local complication rate of $0.17 \%$.

\section{Further analysis}

There appears to be a significantly greater yield of ICA identification (and a resultant alteration of the intervention site) associated with image-guided pleural biopsy procedures. We were intrigued to look into the possible reasons behind this. In our practice, image-guided biopsies are routinely carried out in the lateral decubitus position (similar to medical thoracoscopy and indwelling pleural catheter insertion) and we hypothesised that this may be related to the fact that these patients will often have pleural thickening as a pre-requisite for the procedure to be considered (this would be specifically examined for, either via TUS in the pleural clinic or through their cross-sectional imaging). Increased identification/intervention may be related to the rib crowding often seen in patients with pleural disease, due to loss of lung volume, or it may simply be that pleural thickening slightly alters the anatomy of the vessel, making it more superficially visible.

\section{Pleural thickening}

As the assessment of pleural thickening is not consistently recorded in our procedure reporting proformas, we attempted to assess this further. We carried out a subgroup analysis of presence versus absence of pleural thickening on the cohort of patients who underwent either diagnostic or therapeutic aspiration $(n=310)$. These patients were chosen specifically as they would be more likely to be having the procedure as an initial diagnostic/therapeutic intervention and would therefore be likely to encompass all aetiologies of pleural disease without a pre-requisite for pleural thickening. Two independent researchers (M. Hassan and D.J. McCracken) interrogated the imaging of this aspiration cohort and picked out the first 50 patients who had undergone a contrast-enhanced CT scan of the chest prior to their procedure and who had radiologist-reported parietal pleural thickening. These cases were separated into a "pleural thickening" group and were then matched with 50 cases from the same aspiration cohort to make up a "no pleural thickening" group. This latter group also had to have had a chest CT scan with contrast prior to the procedure but either had radiologist-confirmed absence of pleural thickening or, in the event of no comment on pleural thickening, absence had to be confirmed by two different independent researchers (R. Asciak and R.M. Mercer). Both groups were then matched with their ICA identification data (see table 5). This demonstrated that an ICA was

TABLE 5 Intercostal artery (ICA) identification in relation to pleural thickening

Pleural thickening

No pleural thickening

ICA identified

ICA not identified

31

19
14

36 
identified in 31 out of 50 cases (62\%) with pleural thickening versus 14 out of 50 cases $(28 \%)$ in the absence of pleural thickening (Chi-squared: 11.68 (two degrees of freedom), $\mathrm{p}<0.01$ ).

\section{Discussion}

To the best of the authors' knowledge, this is the first prospective "real life" study of ICA assessment and identification in patients about to undergo pleural procedures (using the largest prospective database from a single centre). A previous study has demonstrated that physicians performing ultrasound can accurately screen the vulnerable ICA [12] but it is noteworthy that the patients in that study did not have pleural disease.

This study demonstrates that chest physicians can identify the ICA without any additional radiology or higher-level ultrasound training. This technique resulted in an alteration of the site of intervention in $15.6 \%$ of screened cases, increasing up to almost one third of cases when the ICA was successfully identified and rarely led to abandonment of procedures altogether. Older age was not shown to increase the chances of identifying the ICA and therefore ICA screening would be encouraged prior to pleural procedures in patients of all ages. The ICA was identified in all procedures conducted through a posterior approach, highlighting the known importance of site consideration with regard to bleeding risk. Additionally, no additional transducers or advanced ultrasound equipment are required to carry this out effectively and, as described above, this step adds no longer than $2 \mathrm{~min}$ to the total procedure time. The increased rate of identification in medical thoracoscopy may be due to these patients often having more significant pleural disease and hence pleural thickening (as in the image-guided biopsy cohort). With regard to diagnostic aspirations, this may be due to an increased frequency of a posterior approach or due to targeting smaller effusions.

Our results do show that there are instances where the site is not altered even when the ICA is identified. This is often related to whether or not the ICA is seen across the whole field of view (e.g. in cases where the ICA is seen on the opposite side to that of intended needle insertion, the operator may deem it safe to proceed as planned). In some cases, adjustment of the probe angle or altering needle angle can also help to avoid the ICA. It should be noted that operators used the most lateral position possible for intervention, as per guidelines on pleural intervention. Despite this procedure practice, the significant number of ICAs detected in the procedure field is therefore a stronger result than that seen in a simple screening study.

Pleural thickening is defined as a focal echogenic lesion with or without an irregular margin, arising from the visceral or parietal pleura, that is greater than $3 \mathrm{~mm}$ in width [15]. This finding appears to increase the chances of identifying the intercostal vessels, although the mechanism for this is not clear. We hypothesise that this may be related to rib crowding often seen in patients with pleural disease due to loss of lung volume or it may simply be that pleural thickening slightly alters the anatomy of the vessel, making it superficially more visible.

The optimal method of assessing vasculature is using a linear high frequency probe, due to the higher sensitivity, but this would require additional training and not be applicable to the majority of physicians regularly engaged in pleural practice. Most studies on intercostal vessel visualisation have used high frequency linear probes, but these were not conducted specifically in patients with pleural disease $[12,16]$ where it is convention to use low frequency curvilinear probes to evaluate and guide intervention. Therefore, one of the strengths of this "real world" study is the intentional use of a low frequency probe to make it generalisable and, by not having to switch probes, to save time. This becomes crucial for the integration of this step into standard pleural practice.

There are some limitations to this study. A range of operators with inevitably varying levels of expertise performed the study; however, by analysing two different time points and comparing the data we demonstrated a similar population, case mix and procedure volume undertaken by our service (approximately 35 procedures per month), as well as consistency across different operators (taking into account fellow/trainee rotations). We believe this adds to the external validity of the results and provides a large number of cases on which to base conclusions. It should be clear, however, that such studies require replication in a non-tertiary centre and with a range of ultrasound equipment. An additional issue is with cases where the ICA is not detected at ultrasound, as we assume that this relates to the artery being truly shielded by the rib above (as would be expected anatomically); however, a false negative scan is another possibility. That said, the lack of pleural bleeding outcomes in this study provides some reassurance against this potential limitation.

\section{Conclusion}

Respiratory physicians with no formal radiology training are able to detect the ICA using the same low frequency transducer used in routine pleural practice and as part of the same pre-procedure planning TUS 
scan. This has been demonstrated in over half of the procedures carried out in this study. While these prospective observational data were not designed to assess the clinical benefit of ICA screening, one would envisage that there is the potential for a safety benefit but this would need a much larger, multicentre cohort given the low incidence of major complications. However, it is noteworthy that there is little published data on such complications and this study shows our unit's complication rate $(0.17 \%)$ to be lower than that in published case series $(2 \%)[17,18]$.

The growth and development of pleural services worldwide is seeing an increasing number of procedures being carried out by physicians, often in ambulatory and outpatient settings. Moreso, with greater experience, procedures of increasing complexity are being conducted in more diverse populations. Patient safety remains at the core of our practice and every effort should be made to enhance this. The premise implied by this study requires further large scale prospective research using a robustly designed study to be truly practice-changing, but nonetheless has suggested that this simple yet important step requires serious consideration in future practice recommendations.

Author contributions: E.O. Bedawi, A. Talwar and N.M. Rahman conceived the study. E.O. Bedawi, A. Talwar, M. Hassan, D.J. McCracken, R. Asciak, R.M. Mercer, R.J. Hallifax and N.I. Kanellakis collected, combined and analysed the data. E.O. Bedawi wrote the first manuscript. F.V. Gleeson, J.M. Wrightson and N.M. Rahman critically reviewed the manuscript. All authors reviewed and approved the final manuscript.

Conflict of interest: None declared.

Support statement: M. Hassan is a recipient of a European Respiratory Society long-term research fellowship (ERS 2016 7333). N.I. Kanellakis and N.M. Rahman are funded by the Oxford NIHR Biomedical Research Centre. Funding information for this article has been deposited with the Crossref Funder Registry.

\section{References}

1 Havelock T, Teoh R, Laws D, et al. Pleural procedures and thoracic ultrasound: British Thoracic Society pleural disease guideline 2010. Thorax 2010; 65: i61-i76.

2 Rahman NM, Singanayagam A, Davies HE, et al. Diagnostic accuracy, safety and utilisation of respiratory physician-delivered thoracic ultrasound. Thorax 2010; 65: 449-453.

3 Psallidas I, Helm EJ, Maskell NA, et al. Iatrogenic injury to the intercostal artery: aetiology, diagnosis and therapeutic intervention. Thorax 2015; 70: 802-804.

4 Kanai M, Sekiguchi H. Avoiding vessel laceration in thoracentesis: a role of vascular ultrasound with color Doppler. Chest 2015; 147: e5-e7.

5 Hernandez MC, Zeb MH, Heller SF, et al. Tube thoracostomy complications increase cost. World J Surg 2017; 41: 1482-1487.

6 Menegozzo CAM, Utiyama EM. Steering the wheel towards the standard of care: proposal of a step-by-step ultrasound-guided emergency chest tube drainage and literature review. Int J Surg Lond Engl 2018; 56: 315-319.

7 Kong VY, Clarke DL. The spectrum of visceral injuries secondary to misplaced intercostal chest drains: experience from a high volume trauma service in South Africa. Injury 2014; 45: 1435-1439.

8 Curtin JJ, Goodman LR, Quebbeman EJ, et al. Complications after emergency tube thoracostomy: assessment with CT. Radiology 1996; 198: 19.

9 Harris A, O'Driscoll BR, Turkington PM. Survey of major complications of intercostal chest drain insertion in the UK. Postgrad Med J 2010; 86: 68-72.

10 Koyanagi T, Kawaharada N, Kurimoto Y, et al. Examination of intercostal arteries with transthoracic Doppler sonography. Echocardiogr 2010; 27: 17-20.

11 Görg C, Bert T, Görg K, et al. Colour Doppler ultrasound mapping of chest wall lesions. Br J Radiol 2005; 78: 303-307.

12 Salamonsen M, Dobeli K, McGrath D, et al. Physician-performed ultrasound can accurately screen for a vulnerable intercostal artery prior to chest drainage procedures. Respirology 2013; 18: 942-947.

13 Helm EJ, Rahman NM, Talakoub O, et al. Course and variation of the intercostal artery by CT scan. Chest 2013; 143: 634-639.

14 Talwar A, Corcoran J, Hallifax R, et al. P4 A prospective assessment of the clinical utility of intercostal artery identification in pleural intervention. Thorax 2016; 71: A84-A85.

15 Koegelenberg CFN, Diacon AH. Radiology: pleural ultrasound. In: Lee YCG, Light RW, eds. Textbook of pleural diseases. 3rd Edn. Boca Raton, CRC Press, 2016; pp. 222-233.

16 Salamonsen M, Ellis S, Paul E, et al. Thoracic ultrasound demonstrates variable location of the intercostal artery. Respir Int Rev Thorac Dis 2012; 83: 323-329.

17 Horsley A, Jones L, White J, et al. Efficacy and complications of small-bore, wire-guided chest drains. Chest 2006 130: $1857-1863$.

18 Hooper C, Maskell N, BTS audit team. British Thoracic Society national pleural procedures audit 2010. Thorax 2011; 66: 636-637. 